\title{
The prevalence of lower eyelid epiblepharon and its association with refractive errors in Chinese preschool children: a cross-sectional study
}

\author{
Deyi Zhuo ${ }^{1,2+}$, Si Chen ${ }^{3+}$, Xiaofang Ren', Bingsong Wang ${ }^{1,4}$, Linbo Liu ${ }^{3,5}$ and Lin Xiao ${ }^{1 *}$ (1)
}

\begin{abstract}
Background: To assess the prevalence and demographics of lower eyelid epiblepharon in Chinese preschool children and to evaluate its association with refractive errors.

Methods: In this population-based, cross-sectional study, a total of 3170 children aged 3 to 6 years from Beijing, China underwent examinations including weight, height, cycloplegic autorefraction and slit-lamp examination of external eyes. The prevalence of lower eyelid epiblepharon in preschool children was evaluated and its association with age, sex, body mass index (BMI), and refractive errors was analyzed using logistic regression analysis.

Results: The prevalence of lower eyelid epiblepharon was $26.2 \%$, which decreased with age, with prevalence in 3-, 4-, 5-, and 6-year-olds of 30.6, 28.0, 15.0, and 14.3\%, respectively. Boys had a higher risk of having epiblepharon than girls $(\mathrm{OR}=1.41 ; 95 \% \mathrm{Cl},(1.20-1.66))$ and no significant correlation was detected between $\mathrm{BMI}$ and epiblepharon after adjusting for age and sex $(p=0.062)$. Epiblepharon was significantly associated with a higher risk of refractive errors, including astigmatism $(\mathrm{OR}=3.41 ; 95 \% \mathrm{Cl},(2.68-4.33))$, myopia $(\mathrm{OR}=3.55 ; 95 \% \mathrm{Cl},(1.86-6.76))$, and hyperopia $(\mathrm{OR}=$ $1.53 ; 95 \% \mathrm{Cl},(1.18-1.99))$.

Conclusions: There is a high prevalence of lower eyelid epiblepharon in Chinese preschool children, particularly among boys and younger children. Preschoolers with lower eyelid epiblepharon are subject to a higher risk of developing astigmatism, myopia, and hyperopia, than those without. Increased attention should be paid to this eyelid abnormality in the preschool population.
\end{abstract}

Keywords: Lower eyelid epiblepharon, Astigmatism, Myopia, Refractive error, Preschool children

\footnotetext{
* Correspondence: xiaolin1957@yeah.net

${ }^{+}$Deyi Zhuo and Si Chen contributed equally to this work.

'Department of Ophthalmology, Beijing Shijitan Hospital, Capital University of Medical Sciences, Beijing 100143, People's Republic of China

Full list of author information is available at the end of the article
}

(c) The Author(s). 2021 Open Access This article is licensed under a Creative Commons Attribution 4.0 International License, which permits use, sharing, adaptation, distribution and reproduction in any medium or format, as long as you give appropriate credit to the original author(s) and the source, provide a link to the Creative Commons licence, and indicate if changes were made. The images or other third party material in this article are included in the article's Creative Commons. licence, unless indicated otherwise in a credit line to the material. If material is not included in the article's Creative Commons licence and your intended use is not permitted by statutory regulation or exceeds the permitted use, you will need to obtain permission directly from the copyright holder. To view a copy of this licence, visit http://creativecommons.org/licenses/by/4.0/ The Creative Commons Public Domain Dedication waiver (http://creativecommons.org/publicdomain/zero/1.0/) applies to the data made available in this article, unless otherwise stated in a credit line to the data. 


\section{Background}

Epiblepharon is an eyelid disorder characterized by a horizontal skinfold that can overlap the eyelid margin, which results in eyelash brushing against the ocular surface $[1,2]$. This eyelid anomaly usually affects the lower eyelid bilaterally and is reported to be common among East Asian descendants [3]. Although it more commonly involves no or mild symptoms, there are still considerable numbers of patients subject to epiblepharon-related discomforts such as tearing, irritation, and photophobia combined with keratopathy given the ever-increasing Asian populations worldwide [4-6].

Several researchers have attempted to document its prevalence and demographic or clinical characteristics in Asian populations including but not limited to Japanese $[3,7]$, Korean [8-10], and Chinese populations [4, 5, 11, 12]. However, to the best of our knowledge, its prevalence in Chinese preschool children is currently unknown. In addition, although the correlation between epiblepharon and refractive errors particularly astigmatism has been previously evaluated [4, 5, 8-12], this association has yet to be assessed in the general population since previous researchers retrospectively reviewed clinical data exclusively from epiblepharon patients who sought medical or surgical treatment at local hospitals [4, 5, 8-12].

In this study, we conducted a population-based study with the aim of exploring the prevalence and demographic characteristics of lower eyelid epiblepharon in Chinese preschool children and assessing its correlation with refractive errors including astigmatism, myopia, and hyperopia.

\section{Methods}

\section{Subjects}

This was a cross-sectional, school-based study of lower eyelid epiblepharon conducted from July to September 2017 in Xicheng District, a district with approximately 20,000 kindergartners in Beijing. Two-stage stratified cluster sampling was used to select students for study inclusion. In the first stage, 20 kindergartens were randomly selected from 120 kindergartens in Xicheng District. In the second stage, all preschoolers from the 20 kindergartens were selected to undergo health examination at Xicheng Maternal and Child Health Care Hospital. The sample size was calculated to be 3458 with a prevalence rate of $10 \%$, a $1 \%$ error rate and a $95 \%$ confidence interval based on the study performed by Noda S [3]. A total of 3721 children aged 3-6 years participated in the study and 3170 of them were included in the data analysis, yielding a completion rate of $85.2 \%$. All participants and their parents or guardians were given full knowledge of the study and written informed consent was obtained from at least one of their parents or guardians.

\section{Physical and eye examinations}

All examinations were conducted at room temperature $\left(\sim 26-28^{\circ} \mathrm{C}\right)$. The height of the children was measured in meters without shoes, and the weight was measured in kilograms. BMI was calculated as weight in kilograms divided by the square of height in meters.

All participants underwent external-eye examination of both eyes by the same ophthalmologist (ZD) using a slit-lamp biomicroscope. Photographs of both eyes were taken using a digital camera against a white background for further diagnosis and classification of epiblepharon. Refractive error was determined by cycloplegic refraction performed with a handheld autorefractor (SureSight ${ }^{\oplus}$ Autorefractor, Welch Allyn) $\geq 30$ mins after cycloplegia which was induced by 3 drops of $1 \%$ cyclopentolate (Cyclogyl, Alcon, Belgium) with an interval of 10 mins. For quality control, the autorefractor was calibrated every day prior to data collection and approximately 5\% of the children were randomly selected to repeat the refraction test.

\section{Definitions of lower eyelid epiblepharon}

Lower eyelid epiblepharon is diagnosed as a redundant skinfold in the lower eyelid with inverted eyelashes touching the corneal surface, but no inward rotation of the eyelid margin [3]. According to Khwarg's classification, the severity of skinfold was categorized into 4 classes according to its height and the degree to which it concealed the eyelid margin in the primary eye position [8]; the severity of cilia-cornea touch was classified into 3 classes according to the area of inverted cilia touching the ocular surface in the primary position (Fig. 1) [8].The children were thereafter considered to have mild epiblepharon if they had any signs of class I, moderate epiblepharon if they demonstrated any signs of class II, and severe epiblepharon if they presented any signs of class III or worse [13]. The diagnosis of lower eyelid epiblepharon was performed independently by two authors (ZD and CS) where disagreement was resolved by discussion with a senior specialist (XL).

\section{Definitions of myopia, hyperopia and astigmatism}

The spherical equivalent (SE) refractive error was expressed as sphere power $+1 / 2 \times$ cylinder power. Myopia was defined as $\mathrm{SE} \leq-0.50$ diopter (D), and hyperopia was defined as $\mathrm{SE} \geq+2.00 \mathrm{D}$. Emmetropia was defined as $\mathrm{SE}$ between $-0.50 \mathrm{D}$ and $+1.00 \mathrm{D}$, non-inclusive. Astigmatism was defined as a cylindrical refractive error (CYL) of at least $1.50 \mathrm{D}$ with cylindrical refractive error presented as negative correcting cylinder form and was classified into three categories, i.e., with-the-rule astigmatism (WTR; cylinder axis between $1^{\circ}$ and $15^{\circ}$ or $165^{\circ}$ and $180^{\circ}$ ), against-the-rule astigmatism (ATR; cylinder axis between $75^{\circ}$ to $105^{\circ}$ ), and oblique astigmatism (OBL; cylinder axis 


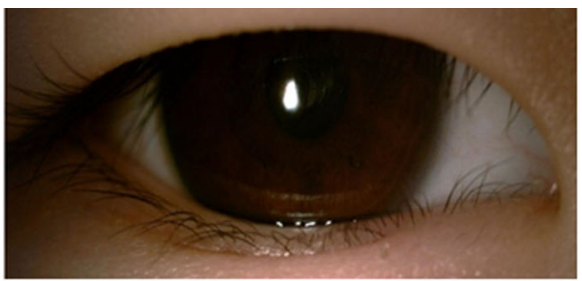

Class I skin fold, Class I cilia touching

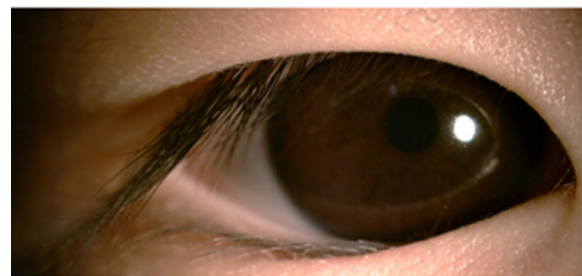

Class III skin fold, Class II cilia touching

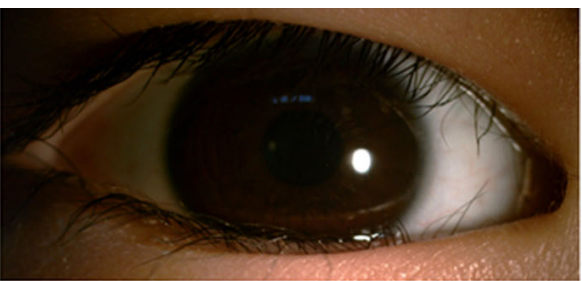

Class II skin fold, Class I cilia touching

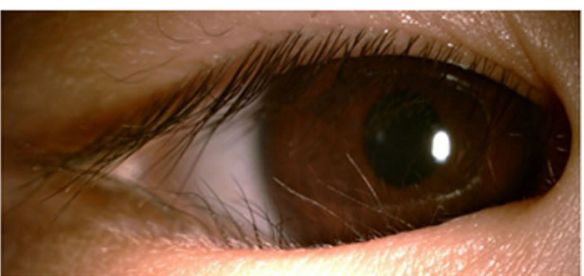

Class V skin fold, Class III cilia touching

Fig. 1 Classification of lower eyelid epiblepharon

between $16^{\circ}$ and $74^{\circ}$ or $106^{\circ}$ and $\left.164^{\circ}\right)$. For children with lower eyelid epiblepharon, if both eyes had equal severity of this condition, refractive error of the right eye was used for analysis; otherwise, the worse eye was selected. For those without epiblepharon, if both eyes had equivalent refractive error, data from the right eye were used for analysis; otherwise, the worse eye was used.

\section{Statistical analysis}

All the data were imported to Epi-Info version 7.0.9.7 for double-entry customizing, and any differences were resolved by checking with the original data. Chi-square test was employed to analyze the differences in sex, age, and refractive errors between children with and without lower eyelid epiblepharon. Independent samples $\mathrm{t}$-tests were used to compare the differences in height, weight, and BMI between children with and without lower eyelid epiblepharon. One-way analysis of variance (ANOVA) was used to assess the differences in the cylindrical power among astigmatic children with different severities of epiblepharon. Multivariable logistic regression analysis was used to evaluate the factors associated with lower eyelid epiblepharon, and odds ratios (ORs) and the 95\% confidence intervals (CIs) were calculated. All analyses were performed using SPSS (version 22, SPSS Inc., Chicago, Illinois), and a two-sided $p<0.05$ was considered to be statistically significant.

\section{Results}

There were 1652 boys and 1518 girls aged 3-6 years included in the analysis and 551 children were excluded owing to incompletion of the study $(n=548)$, true entropion $(n=1)$ and external hordeolum $(n=2)$. There was no significant difference in age between boys $(4.10 \pm 0.75 \mathrm{yrs})$ and girls $(4.08 \pm 0.76 \mathrm{yrs})$ of all included subjects (independent samples t-test; $p=0.46$ ). Overall, 26.2\% (830 out of 3170) children had lower eyelid epiblepharon, which could be further grouped into mild (54.3\%), moderate (29.6\%) and severe (16.1\%) classes based on the severity of skin-fold height and cilia-corneal touch (Table 1).

Table 2 summarizes the general profile of all included children. Statistically significant differences were detected in sex, age, $\mathrm{SE}, \mathrm{CYL}$, astigmatism type (chi-square tests; $p=<0.001,<0.001,<0.001,<0.001$, and 0.022,

Table 1 Classification of lower eyelid epiblepharon

\begin{tabular}{llllll}
\hline $\begin{array}{l}\text { Class of } \\
\text { cilia-cornea } \\
\text { touching }\end{array}$ & \multicolumn{2}{l}{ Class of skin fold height } & III & IV & Total \\
\cline { 2 - 5 } & I & II & $1(0.1 \%)^{c}$ & $0(0 \%)^{c}$ & \\
\hline I & $451(54.3 \%)^{a}$ & $84(10.1 \%)^{b}$ & $66(8.0 \%)^{c}$ & $1(0.1 \%)^{c}$ & $229(27.6 \%)$ \\
II & $2(0.2 \%)^{b}$ & $160(19.3 \%)^{b}$ & $36(4.3 \%)^{c}$ & $26(3.1 \%)^{c}$ & $65(7.9 \%)$ \\
III & $0(0 \%)^{c}$ & $3(0.5 \%)^{c}$ & $103(12.4 \%)$ & $27(3.2 \%)$ & $830(100 \%)$ \\
Total & $453(54.5 \%)$ & $247(29.9 \%)$ & & \\
\hline
\end{tabular}

${ }^{\mathrm{a}}$ Mild epiblepharon

${ }^{\mathrm{b}}$ Moderate epiblepharon

'Severe epiblepharon 
Table 2 Characteristics of children with and without lower eyelid epiblepharon

\begin{tabular}{|c|c|c|c|}
\hline & $\begin{array}{l}\text { Epiblepharon } \\
(n=830)\end{array}$ & $\begin{array}{l}\text { No epiblepharon } \\
(n=2340)\end{array}$ & $P$ value \\
\hline Gender, n (\%) & & & $<0.001^{*}$ \\
\hline Boys & $487(29.5)$ & $1165(70.5)$ & \\
\hline Girls & $343(22.6)$ & $1175(77.4)$ & \\
\hline Age (yrs), n (\%) & & & $<0.001^{*}$ \\
\hline 3 & $152(30.6)$ & $344(69.4)$ & \\
\hline 4 & $601(28.0)$ & $1547(72.0)$ & \\
\hline 5 & $39(15.0)$ & $221(85.0)$ & \\
\hline 6 & $38(14.3)$ & $228(85.7)$ & \\
\hline SE (D), n (\%) & & & $<0.001^{*}$ \\
\hline$\leq-0.5$ & $22(56.4)$ & $17(43.6)$ & \\
\hline-0.5 to 2 & $713(25.0)$ & $2141(75.0)$ & \\
\hline$\geq 2$ & $95(34.3)$ & $182(65.7)$ & \\
\hline CYL (D), n (\%) & & & $<0.001^{*}$ \\
\hline 0 to $<1$ & $541(22.3)$ & $1890(77.7)$ & \\
\hline 1 to $<1.5$ & $132(30.6)$ & $300(69.4)$ & \\
\hline 1.5 to $<2$ & $68(43.0)$ & $90(57.0)$ & \\
\hline 2 to $<2.5$ & $44(57.1)$ & $33(42.9)$ & \\
\hline 2.5 to $<3$ & $32(65.3)$ & $17(34.7)$ & \\
\hline$>3.0$ & $13(56.5)$ & $10(43.5)$ & \\
\hline Astigmatism type, n (\%) & & & $0.022^{*}$ \\
\hline WTR & $127(52.3)$ & $112(47.4)$ & \\
\hline ATR & $7(35.5)$ & $20(64.5)$ & \\
\hline OBL & $23(56.1)$ & $18(43.9)$ & \\
\hline Height (ms), mean (SD) & $1.01(0.07)$ & $1.01(0.07)$ & 0.303 \\
\hline Weight (kgs), mean (SD) & $15.72(2.92)$ & $15.63(2.73)$ & 0.413 \\
\hline BMI, mean (SD) & $15.43(1.83)$ & $15.26(1.69)$ & $0.018^{*}$ \\
\hline
\end{tabular}

$B M I$ Body mass index, SD Standard deviation, CI Confidence interval, SE Spherical equivalent refractive error, CYL Cylindrical refractive error, D Diopters, WTR Withthe-rule, ATR Against-the-rule, $O B L$ Oblique

${ }^{*} p$ is statistically significant at $5 \%$

respectively) and BMI (independent samples t-test; $p=0.018$ ) between epiblepharon and nonepiblepharon children (Table 2); no significant differences were identified in either height or weight between these two groups (independent samples t-test; $p=0.303$ and 0.413 , respectively) (Table 2 ).

The prevalence of lower eyelid epiblepharon based on age was 30.6, 28.0, 15.0, and 14.3\% for 3-, 4-, 5-, and 6year-old children, respectively (Table 2). Figure 2 further illustrates the age-specific prevalence of lower eyelid epiblepharon by its severity according to criteria based on skinfold height only (Fig. 2a), cilia-cornea touching area only (Fig. 2b), and criteria established by both (Fig. 2c). At different degrees of severity, younger patients roughly demonstrated a higher prevalence of epiblepharon than older patients (Fig. 2).

\section{Lower eyelid epiblepharon associated risk factors}

As statistically significant differences in age, sex and BMI were detected between children with and without lower eyelid epiblepharon (Table 2), multivariable logistic regression analysis was performed to further evaluate the association between lower eyelid epiblepharon and these three variables to screen for the risk factors for this eyelid disorder (Table 3). After adjustment for potential confounders including age, sex and BMI, epiblepharon was significantly associated with sex and age. Specifically, boys were more likely to have epiblepharon than girls $(\mathrm{OR}=$ 1.41 with $p<0.001$ ); younger children at 3,4 , and 5 years old demonstrated higher possibilities of having epiblepharon than older children (6 years old), although no statistically significant difference was identified between 5 - and 6-year-old children $(\mathrm{OR}=3.68,2.95$, and 1.24 with $p=<$ 

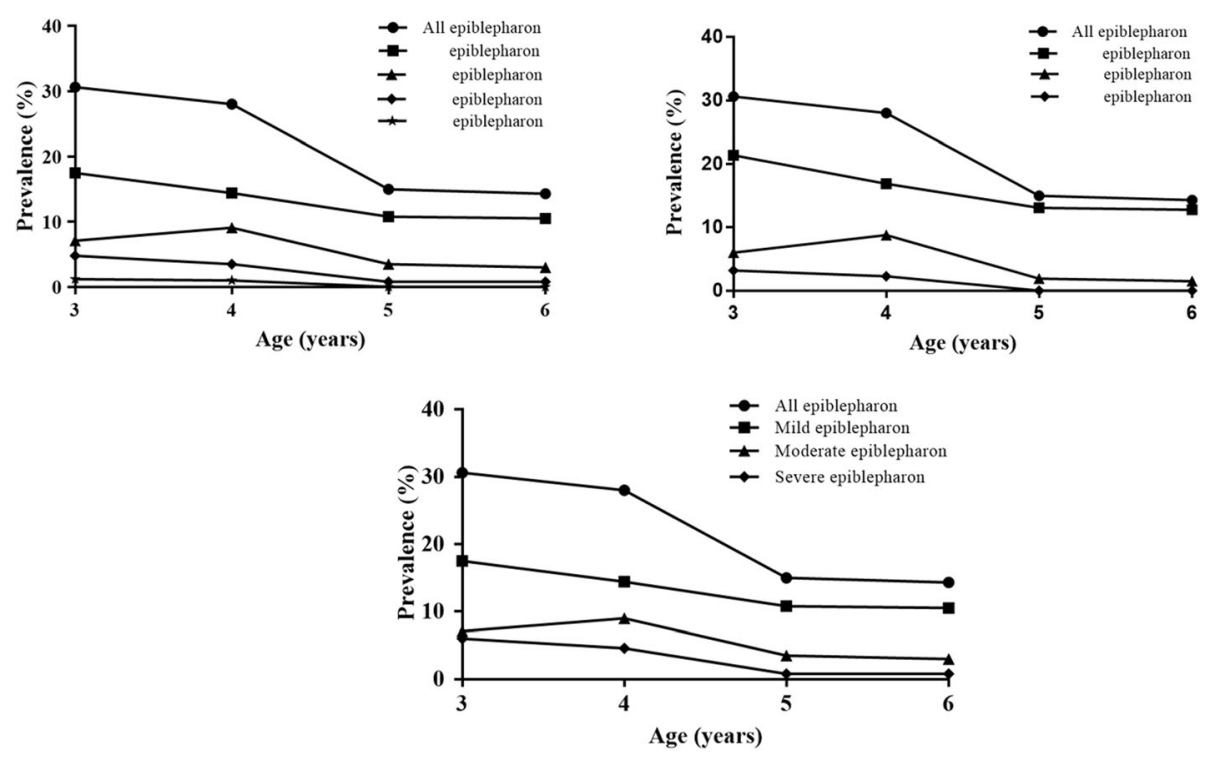

Fig. 2 Age-specific prevalence of lower eyelid epiblepharon by its severity. Epiblepharon classified by skinfold height (a), cilia-cornea touching area (b), and both skinfold height and cilia-cornea touching area (c)

$0.001,<0.001$ and 0.402, respectively). However, after adjusting for sex and age, the association between BMI and epiblepharon was no longer statistically significant $(p=0.062)$.

\section{Relation between lower eyelid epiblepharon and refractive errors}

Astigmatism (CYL $\geq 1.5$ D) was identified in 307 children (9.68\%) and 157 of them had epiblepharon. A statistically significantly higher prevalence of astigmatism was detected in epiblepharon children than in nonepiblepharon children (18.9\% vs 6.4\%) (chi-square test; $p<0.001)$. Meanwhile, in both populations, the astigmatism type was largely WTR $(80.9 \%$ for epiblepharon vs $74.7 \%$ for nonepiblepharon).

Myopia ( $\mathrm{SE} \leq-0.5 \mathrm{D})$ was recognized in 39 children (1.23\%) with 22 of them having epiblepharon; hyperopia ( $\mathrm{SE} \geq 2 \mathrm{D}$ ) was found in 277 children (8.74\%) with 95 of them being epiblepharon-positive. Higher prevalences of

Table 3 Association between lower eyelid epiblepharon and age, gender, and BMI

\begin{tabular}{llll}
\hline Risk factors & OR & $\mathbf{9 5 \% C l}$ & $P$ value \\
\hline $\begin{array}{l}\text { Gender } \\
\text { Boys vs girls }\end{array}$ & 1.41 & $(1.20,1.66)$ & $P<0.001$ \\
$\begin{array}{l}\text { Age (yrs) } \\
\mathbf{3} \text { vs } \mathbf{6}\end{array}$ & 3.68 & $(2.38,5.68)$ & $P<0.001$ \\
$\mathbf{4}$ vs $\mathbf{6}$ & 2.95 & $(2.01,4.32)$ & $p<0.001$ \\
$\mathbf{5}$ vs $\mathbf{6}$ & 1.24 & $(0.75,2.03)$ & $p=0.402$ \\
BMI & 1.07 & $(0.95,1.15)$ & $P=0.062$ \\
\hline
\end{tabular}

OR Odds ratio, $\mathrm{Cl}$ Confidential interval myopia (2.7\% vs $0.7 \%$ ) and hyperopia ( $11.4 \%$ vs $7.8 \%)$ were identified in epiblepharon children than in nonepiblepharon children (chi-square tests; $p=<0.01$ and 0.002 , respectively). To further evaluate the association between lower eyelid epiblepharon and refractive errors, multivariable logistic regression analysis was performed where age and sex were adjusted for (Table 4). Overall, epiblepharon children presented an increased risk of astigmatism relative to nonepiblepharon children $(\mathrm{OR}=3.41 ; 95 \% \mathrm{CI}$, $(2.68,4.33))$, and epiblepharon preschoolers had a higher risk of myopia $(\mathrm{OR}=3.55$; 95\% $\mathrm{CI},(1.86,6.76))$ and hyperopia $(\mathrm{OR}=1.5395 \% \mathrm{CI},(1.18,1.99))$ than nonepiblepharon children.

\section{Discussion}

This population-based cross-sectional study investigated the prevalence and demographics of lower eyelid epiblepharon and explored its correlation with refractive errors in 3- to 6-year-old Chinese children. To the best of our knowledge, our study is the first to reveal the prevalence of this eyelid disorder in Chinese preschoolers and analyzes the relationship between epiblepharon and refractive errors including not only astigmatism but also myopia and hyperopia. We observed a prevalence of $26.2 \%$ in 3 - to 6year-old Chinese children, much higher than that of Noda S. et al.'s study in Japanese children, where $13.8 \%$ of 766 children were diagnosed with epiblepharon [3]. The relatively lower prevalence in Noda's study is possibly because they adopt relatively stricter criteria for disease diagnosis where the widely accepted Khwarg's classification has yet to be established. In Noda's study, cilia-cornea touching was confirmed only when the cornea was positively 
Table 4 Association between lower eyelid epiblepharon and refractive errors

\begin{tabular}{|c|c|c|c|c|c|c|}
\hline & \multicolumn{2}{|c|}{ Astigmatism( $\geq 1.5 \mathrm{D})$} & \multicolumn{2}{|c|}{ Myopia( $\leq-0.5 \mathrm{D})$} & \multicolumn{2}{|c|}{ Hyperopia( $\geq 2.0 \mathrm{D})$} \\
\hline & OR $(95 \% \mathrm{Cl})$ & $P$ value & OR $(95 \% \mathrm{Cl})$ & $P$ value & OR $(95 \% \mathrm{Cl})$ & $P$ value \\
\hline No Epiblepharon & 1.00 (ref) & & 1.00 (ref) & & 1.00 (ref) & \\
\hline \multicolumn{7}{|l|}{ Epiblepharon } \\
\hline Mild & $3.15(2.35,4.22)$ & $<0.001$ & $4.38(2.14,8.95)$ & $<0.001$ & $1.51(1.09,2.10)$ & 0.014 \\
\hline Moderate & $3.82(2.69,5.42)$ & $<0.001$ & $4.00(1.64,9.75)$ & 0.002 & $1.52(1.00,2.32)$ & 0.050 \\
\hline Severe & $3.55(2.24,5.62)$ & $<0.001$ & - & - & $1.62(0.94,2.79)$ & 0.082 \\
\hline All & $3.41(2.68,4.33)$ & $<0.001$ & $3.55(1.86,6.76)$ & $<0.001$ & $1.53(1.18,1.99)$ & 0.001 \\
\hline
\end{tabular}

OR Odd ratio, WTR With the rule, OBL Oblique

stained after topical fluorescein staining, whereas later studies by Khwang S. et al. and Young S. et al. indicated that a considerable number of epiblepharon patients (usually those with mild epiblepharon) may not have the ocular manifestation of corneal erosion that could be stained with fluorescein $[8,13]$. In addition, when we excluded children with mild epiblepharon, the prevalence decreased to $11.9 \%$, which was similar to the $13.8 \%$ reported in Noda's study.

Given such a high prevalence in Asian infants and children, deducing the demographic characteristics of epiblepharon is helpful for a better understanding of this eyelid disorder. To date, limited data are available from either observations of the general population [3, 7] or retrospective data from patients who underwent surgical treatment [4]. Consistent with previous studies [3, 4, 7], our results in Chinese preschool children show that younger individuals have a higher risk of epiblepharon, supporting the widely-accepted hypothesis that epiblepharon tends to disappear spontaneously with aging as facial bone growth in Asians [6]. However, there are discrepancies in BMI and the sexual predilection between our study and others. Different from results in Japanese and Singaporeans where no sexual predilection was recognized $[3,4,7]$, the present study demonstrates that boys are subject to a higher risk of having lower eyelid epiblepharon than girls with an adjusted OR $=1.41$ in the logistic regression model. Differences in the diagnostic criteria [3], subjects' age range [7], epiblepharon severity, and possible sample selection bias in a clinic-based study [4] may all contribute to the discrepancy in sex predilection between our study and others.

Although lower eyelid epiblepharon trends to resolve spontaneously with aging, its occurrence during the critical period of visual development makes it crucial to disclose if it is correlated with refractive errors, which may ultimately lead to permanent visual impairment such as amblyopia or retinopathy [14-17]. In this study, the prevalence of astigmatism was $9.68 \%$, similar to previous studies in Asian infants and preschoolers [18-21], and, in those with lower eyelid epiblepharon, the prevalence could be as high as $18.9 \%$. We demonstrate for the first time that epiblepharon children are subject to a significantly higher risk of astigmatism, largely being WTR in the general population, and these findings agree well with previous retrospective studies in patients $[9,11,12]$. In addition, we also evaluated the relationship between lower eyelid epiblepharon and spherical refractive errors, i.e., myopia and hyperopia. These epiblepharon preschoolers were 3.55 times as likely to have myopia as those without epiblepharon, and 1.53 times as likely to have hyperopia. The significantly increased risk of myopia in epiblepharon children agrees with the observations in clinical patients that severe myopia is commonly accompanied by a large number of cilia touching the cornea [9]. However, considering the relatively small sample size (22 out of 39 epiblepharon preschoolers being myopic) and absence of severe myopia ($2.75 \mathrm{D}$ to $-0.5 \mathrm{D}$ ), further study with a larger sample size should be conducted to further validate the association between epiblepharon and myopia.

The strength of this study lies in the fact that the demographics of epiblepharon and its association with refractive errors are evaluated in the Chinese preschool population with by far the largest sample size thus far reported. Nevertheless, there are several limitations in this study. First, the children included in this study were all enrolled from urban areas, which may result in bias from sample selection. Further studies ideally from multiple centers including children from both rural and urban area would improve our understanding of the risk factors related to lower eyelid epiblepharon and its association with refractive errors. Second, the prevalence of epiblepharon may be underestimated because epiblepharon can be missed in children whose cilia touch the cornea only in downward gaze but not in primary gaze. Another limitation is that we used only the skinfold height and the cilia-cornea touching area as criteria for the diagnosis of lower eyelid epiblepharon without considering areas of corneal erosion. However, owing to concerns about the risk of the fluorescein dying procedure and limited time allowance in a screening circumstance, an approach that is both safe and simple must be adopted; in addition, this limitation can be mitigated since there is high agreement among the three criteria for the diagnosis of epiblepharon [8]. In addition, 
our conclusion may be affected by type 1 error as multiple comparisons were conducted.

\section{Conclusions}

our study demonstrates a relatively high prevalence of lower eyelid epiblepharon in Chinese preschoolers, particularly in boys and young children, and shows that there are significant correlations between lower eyelid epiblepharon and refractive errors, including astigmatism, myopia, and hyperopia. Given such a high prevalence combined with the increased risk for refractive errors, it would be of great significance to be aware of the necessity and importance to establish an effective screening strategy for this disease, to conduct a closer follow-up of the clinical manifestations of involved children and to consider giving early interventions and visual rehabilitation when warranted.

\section{Abbreviations}

AORs: Adjusted odds ratios; ORs: Odds ratios; Cls: Confidence intervals; BMI: Body mass index; SD: Standard deviation; SE: Spherical equivalent refractive error; CYL: Cylindrical refractive error; WTR: With-the-rule; ATR: Against-the-rule; OBL: Oblique

\section{Acknowledgements}

We thank all subjects for participating in this study.

\section{Authors' contributions}

$X \mathrm{X}$ and $\mathrm{LL}$ designed and supervised this study. ZD, CS and RX collected data. $\mathrm{ZD}, \mathrm{CS}$ and $\mathrm{LL}$ conducted data analysis and interpretation. CS and ZD drafted the manuscript. RX, WB, and XL provided administrative, technical, and material support for this study. All authors have read and approved the manuscript.

\section{Funding}

This study was supported by the Ministry of Education Singapore under its Academic Research Fund Tier 1 (2018-T1-001-144), Agency for Science, Technology and Research (A*STAR) under its Industrial Alignment Fund (Prepositioning) (H17/01/a0/008), National Medical Research Council Singapore under its Open Fund - Individual Research Grant (MOH-OFIRG19may-0009), and Beijing Shijitan Hospital, Capital Medical University under its Hospital Fund (2017-C07). The Funding sponsors were not involved in the study design, data analysis, results interpretation, or manuscript writing.

\section{Availability of data and materials}

The datasets used and/or analyzed during the current study are available from the corresponding author on reasonable request.

\section{Ethics approval and consent to participate}

All procedures conformed to the tenets of the declaration of Helsinki and written informed consent was obtained from the parents or guardians of the participants. The Ethics Committee of Beijing Shijitan Hospital approved the study (2017 Research ethics NO.69, Beijing Shijitan Hospital, Affiliated to Capital Medical University, 2017-5-20).

\section{Consent for publication}

Not applicable.

\section{Competing interests}

No authors have conflict of interest to declare.
Technological University, Singapore 639798, Singapore. ${ }^{4}$ Department of Ophthalmology, Beijing Tongren Hospital, Capital University of Medical Sciences, Beijing 100730, People's Republic of China. ${ }^{5}$ School of Chemical and Biomedical Engineering, Nanyang Technological University, Singapore 637459, Singapore.

Received: 5 August 2020 Accepted: 30 November 2020

Published online: 04 January 2021

\section{References}

1. Johnson CC. Epiblepharon. Am J Ophthalmol. 1968;66(6):1172-5.

2. Lemke BN, Stasior OG. Epiblepharon. An important and often missed diagnosis. Clin Pediatr. 1981;20(10):661-2.

3. Noda S, Hayasaka S, Setogawa T. Epiblepharon with inverted eyelashes in Japanese children. I. Incidence and symptoms. Br J Ophthalmol. 1989:73(2):126-7.

4. Sundar G, Young SM, Tara S, Tan AM, Amrith S. Epiblepharon in East Asian patients: the Singapore experience. Ophthalmology. 2010;117(1):184-9.

5. Yan Y, Chen T, Wei W, Li D. Epiblepharon in Chinese children: relationships with body mass index and surgical treatment. J AAPOS. 2016;20(2):148-52.

6. Kim JS, Jin SW, Hur MC, et al. The clinical characteristics and surgical outcomes of epiblepharon in korean children: a 9-year experience. J Ophthalmol. 2014:2014:156501.

7. Hayasaka Y, Hayasaka S. Epiblepharon with inverted eyelashes and high body mass index in Japanese children. J Pediatr Ophthalmol Strabismus. 2005;42(5):300-3.

8. Khwarg SI, Lee YJ. Epiblepharon of the lower eyelid: classification and association with astigmatism. Korean J Ophthalmol. 1997;11(2):111-7.

9. Yang SW, Choi WC, Kim SY. Refractive changes of congenital entropion and epiblepharon on surgical correction. Korean J Ophthalmol. 2001;15(1):32-7.

10. Ahn HB, Seo JW, Yoo JH, Jeong WJ, Park WC, Rho SH. Epiblepharon related to high body mass index in Korean children. J Pediatr Ophthalmol Strabismus. 2011;48(1):57-60

11. Preechawai P, Amrith S, Wong I, Sundar G. Refractive changes in epiblepharon. Am J Ophthalmol. 2007;143(5):835-9.

12. Shih MH, Huang FC. Astigmatism in children with epiblepharon. Cornea. 2007;26(9):1090-4

13. Young SM, Kim YD, Woo Kl. Modified everting sutures: an alternative treatment for mild to moderate epiblepharon. Br J Ophthalmol. 2018; 102(11):1510-4

14. Woo Kl, Kim YD. Management of epiblepharon: state of the art. Curr Opin Ophthalmol. 2016;27(5):433-8.

15. Xiao O, Guo X, Wang D, et al. Distribution and severity of myopic maculopathy among highly myopic eyes. Invest Ophthalmol Vis Sci. 2018; 59(12):4880-5.

16. Ziylan S, Yabas O, Zorlutuna N, Serin D. Isoametropic amblyopia in highly hyperopic children. Acta Ophthalmol Scand. 2007:85(1):111-3.

17. Xiao O, Morgan IG, Ellwein LB, He M, Refractive Error Study in Children Study G. Prevalence of amblyopia in school-aged children and variations by age, gender, and ethnicity in a multi-country refractive error study. Ophthalmology. 2015;122(9):1924-31.

18. Dirani $M, C h a n ~ Y H$, Gazzard $G$, et al. Prevalence of refractive error in Singaporean Chinese children: the strabismus, amblyopia, and refractive error in young Singaporean Children (STARS) study. Invest Ophthalmol Vis Sci. 2010;51(3):1348-55.

19. Wen G, Tarczy-Hornoch K, McKean-Cowdin R, et al. Prevalence of myopia, hyperopia, and astigmatism in non-Hispanic white and Asian children: multiethnic pediatric eye disease study. Ophthalmology. 2013;120(10):2109-16.

20. Lan $W$, Zhao F, Lin L, et al. Refractive errors in 3-6 year-old Chinese children: a very low prevalence of myopia? PLoS One. 2013:8(10):e78003.

21. Read SA, Collins MJ, Carney LG. A review of astigmatism and its possible genesis. Clin Exp Optom. 2007:90(1):5-19.

\section{Publisher's Note}

Springer Nature remains neutral with regard to jurisdictional claims in published maps and institutional affiliations.

\section{Author details}

'Department of Ophthalmology, Beijing Shijitan Hospital, Capital University of Medical Sciences, Beijing 100143, People's Republic of China. ${ }^{2}$ Department of Ophthalmology, Chinese PLA General Hospital, Beijing 100853, People's Republic of China. ${ }^{3}$ School of Electrical and Electronic Engineering, Nanyang 